\title{
Modal Coupling of Single Photon Emitters Within Nanofiber Waveguides
}

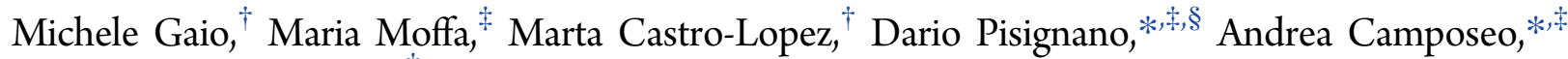
and Riccardo Sapienza* ${ }^{\dagger}$

\author{
${ }^{\dagger}$ Department of Physics, King’s College London, Strand, London WC2R 2LS, United Kingdom \\ ${ }^{\ddagger}$ CNR-Istituto Nanoscienze, Euromediterranean Center for Nanomaterial Modelling and Technology (ECMT), via Arnesano, \\ I-73100 Lecce, Italy \\ ${ }^{\S}$ Dipartimento di Matematica e Fisica “Ennio De Giorgi”, Università del Salento, via Arnesano I-73100 Lecce, Italy
}

\section{Supporting Information}

\begin{abstract}
Nanoscale generation of individual photons in confined geometries is an exciting research field aiming at exploiting localized electromagnetic fields for light manipulation. One of the outstanding challenges of photonic systems combining emitters with nanostructured media is the selective channelling of photons emitted by embedded sources into specific optical modes and their transport at distant locations in integrated systems. Here, we show that soft-matter nanofibers, electrospun with embedded emitters, combine subwavelength field localization and large broadband near-field coupling with low propagation losses. By momentum spectroscopy, we quantify the modal coupling efficiency identifying the regime of single-mode coupling. These nanofibers do not rely on resonant interactions, making them ideal for room-temperature operation, and offer a scalable platform for future quantum information technology.
\end{abstract}

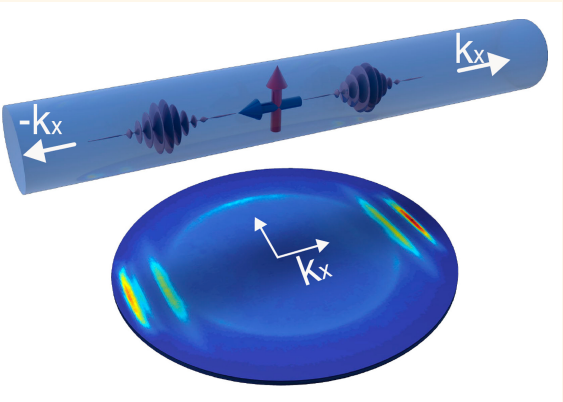

KEYWORDS: nanofiber, nanophotonics, colloidal quantum dots, single photon, momentum spectroscopy, all-dielectric waveguide

\section{$\mathrm{L}$}

ight - matter interaction design in nanostructured optical environments has revolutionized the nanoscale control of light emission, absorption, and transport. Exerting such control on single-photons generated by individual emitters is also a fundamental step toward integrated quantum technologies, with applications including single-photon transistors, ${ }^{1,2}$ on-chip generation and routing of single photons, ${ }^{3-5}$ quantum interference, ${ }^{6,7}$ or long-range coupling of single emitters. $^{8}$

In this framework, plasmonic systems, which exploit nearfield interactions in narrow gaps or nanosized resonators, ${ }^{9,10}$ have attracted much attention for broad-band and room temperature operation: plasmonic nanoantennas can increase the directionality ${ }^{11}$ and decay-rate ${ }^{12,13}$ of single-photon sources, whereas the emitted photons can be transported to distant locations by plasmon modes in metal nanowires, ${ }^{14}$ wedges, ${ }^{15}$ or V-groove metal surfaces. ${ }^{16}$

Inspired by plasmonic architectures, subwavelength dielectric nanostructures are emerging as candidate materials for light management at room-temperature. Unlike photonic crystals, which exploit long-range interference (over many wavelengths) to design narrow resonance modes, subwavelength dielectrics offer practical local field enhancements and the ability of nearfield light manipulation with the advantage of being free of the metal optical losses. ${ }^{17-19}$
Indeed, these waveguides can channel and transport light from a single emitter across long distances with relatively low losses, realizing a simple interface between light emitters and propagating modes, ${ }^{20}$ increasing off-chip extraction efficiency with diamond nanowires ${ }^{21}$ and semiconductor nanopillars. ${ }^{22,23}$ Moreover, the interaction of an emitter with a subwavelength waveguide allows for the exploitation of unique phenomena, such as spin-orbit coupling and unidirectional emission, which occurs only when light is spatially confined. ${ }^{24,25}$

Polymer subwavelength waveguides fabricated by selfassembly, drawn from polymer solutions and melts, ${ }^{26}$ are a promising system compatible with integration of quantum dots or molecules as emitters, and with potential for scalability and interconnection to form networks in 2-dimensions ${ }^{27}$ and 3dimensions. ${ }^{28}$ This improves on conventional pulled silica fibers with emitters evanescently coupled on the nanofiber surface $^{5,29}$ and on pulled capillary hosting single molecules. ${ }^{30}$ Moreover, these waveguides are cavity-free and with a broadband response, which makes them ideal for roomtemperature (where sources are broadband) and ultrafast operation and for further interfacing with biological matter.

Received: March 24, 2016

Accepted: May 20, 2016

Published: May 20, 2016 
Although polymer nanofibers do not require nanolithography or top-down nanofabrication to be realized, the emitterwaveguide coupling has to be assessed a posteriori. In this respect, characterizing the different modal contributions is important for further optical engineering of the propagation and coupling properties, and for quantum applications. The coupling efficiency can be probed from the collected scattered and transmitted light when the waveguide terminals are accessible, ${ }^{5,29}$ by measuring the excitation extinction, ${ }^{30}$ or when coupling to strong resonances from indirect estimations, such as those drawn from the lifetime variations of on- and offcoupling. ${ }^{18,31}$ To date, quantifying the coupling efficiency of a single emitter to a specific nanofiber mode, in a way valid also for subwavelength and weakly resonant modes, is an outstanding challenge.

Here, we report broadband single-photon generation and transport from isolated quantum dots embedded in the core of a free-standing and subwavelength polymer nanofiber. By means of momentum spectroscopy, we record the full $k$-vector distribution of the light emitted by individual quantum dots, revealing the selective coupling of the different dipole orientations to the individual nanofiber modes. We find a coupling to the fundamental mode with values up to $\beta_{01}=(31$ $\pm 2) \%$ which compare well with the theoretical maximum coupling of $\beta_{01}=53 \%$.

\section{RESULTS AND DISCUSSION}

The hybrid nanofiber-emitter system is designed for (i) maximal coupling, by embedding the emitter at the nanofiber core, and (ii) efficient long-range transport, which is achieved by a free-standing geometry. The nanofibers are fabricated by electrospinning a polymethylmetacrylate (PMMA) solution (see Methods) doped with CdSeTe colloidal quantum dots (Invitrogen QD800, emission peaked at $790 \mathrm{~nm}$ ). Unlike electron-beam or focused ion-beam lithography, the electrospinning process largely preserves the integrity of embedded organic and inorganic light emitters. ${ }^{26}$ Doped fibers with a diameter $(D)$ between 300 and $1000 \mathrm{~nm}$, depending on the processing parameters, are deposited either (i) on a glass substrate or (ii) on a transmission electron microscopy (TEM) grid producing free-standing nanostructures, with a circular cross section and length of tens to hundreds of micrometers with isolated quantum dots. A scanning electron microscopy (SEM) image of a typical free-standing nanofiber is shown in Figure 1a. When illuminated with a red laser $(632 \mathrm{~nm})$, the emitters can be located via wide-field fluorescence imaging, as in Figure $1 \mathrm{~b}$. The emission dynamics is studied by confocal microscopy (numerical aperture, $\mathrm{NA}=0.95$ ) and time correlated single-photon counting (TCSPC). Hanbury Brown and Twiss interferometry of the quantum dots in free-standing nanofibers using two avalanche photodiode (APD) in startstop configuration is shown in Figure 1c. The intensity correlation function $g_{2}(t)$ measured under continuous wave (CW) excitation (power $12 \mu \mathrm{W}$, wavelength $633 \mathrm{~nm}$ ) is fitted to $g_{2}(t)=A\left(1-(1 / N) \exp \left(|t| / \tau^{\prime}\right)\right)$, which gives a second-order intensity correlation function at zero-time, $g_{2}(0)=0.10 \pm 0.03$, and a corresponding decay-time $\tau^{\prime}=(84 \pm 3)$ ns, which confirms single-photon emission. The excited state lifetime for the quantum dot is measured under picosecond pulsed excitation (average power $1.5 \mu \mathrm{W}$, repetition rate $2.5 \mathrm{MHz}$, wavelength $634 \mathrm{~nm}$ ). In the specific case shown in Figure 1d, a single exponential fit gives a decay time $\tau=(137 \pm 0.1) \mathrm{ns}$. The difference between $\tau$ and $\tau^{\prime}$ can be explained via an incoherent
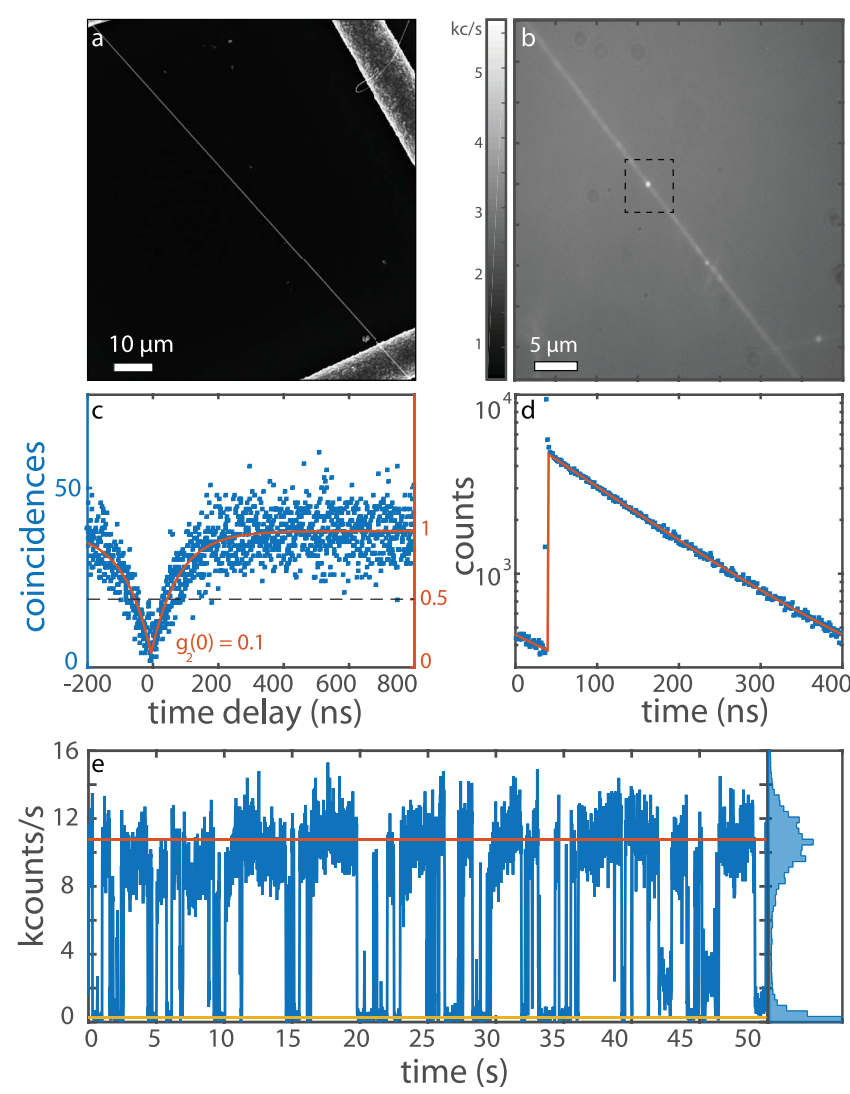

Figure 1. Addressing a single quantum dot coupled to a nanofiber. (a) SEM picture of a free-standing nanofiber. (b) Wide-field fluorescence image of a similar nanofiber highlighting the presence of isolated quantum dots (dashed square). (c) The discrete nature of the photons emitted by the single quantum dot is evidenced by the second order correlation function, obtained via continuouswave laser excitation $(12 \mu \mathrm{W}, 633 \mathrm{~nm})$ which leads to $\mathrm{g}_{2}(0)=0.1$ \pm 0.03 when fitted with $g_{2}(t)=\mathrm{A}\left(1-(1 / N) \exp \left(|t| / \tau^{\prime}\right)\right)$. (d) The fluorescence dynamics of the same quantum dot under picosecond excitation $(1.5 \mu \mathrm{W}, 2.5 \mathrm{MHz}, 634 \mathrm{~nm})$ is fitted with a single exponential decay leading to an excited state lifetime $\tau=(137 \pm$ 0.1) ns. (e) The time trace of the photons emitted by the quantum dot shows a blinking behavior with on- $(\sim 10.5 \mathrm{kcounts} / \mathrm{s})$ and off$(\sim 0.3 \mathrm{kcounts} / \mathrm{s})$ states as highlighted in the histogram.

repumping term $R$, with $\tau^{\prime}=((1 / \tau)+R)^{-1}$, as in ref 32 . Fluorescence lifetime statistics of individual quantum dots inside free-standing nanofibers show a decay rate reduction of $\sim 25 \%$ as compared to a reference homogeneous polymer film, in agreement with the theoretical prediction (Supporting Information Figure S1). The decay rate of an emitter inside a subwavelength nanofiber is expected to be weakly reduced, due to the low refractive index of PMMA $\left(n_{\text {PMMA }}=1.49\right.$, see Supporting Information Figure S1). Light collected from the nanofiber-coupled quantum dots has a typical blinking behavior with an off state of 300 counts/s (Figure 1e).

For a quantum dot in the nanofiber, the quantum dot

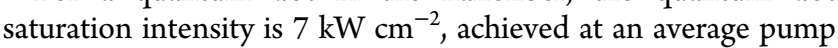
intensity of $0.5 \mu \mathrm{W}$ in a diffraction-limited illumination area (air objective, NA $=0.95)$, for which we have measured 38 kphotons/s emitted, with an expected maximum count-rate $\left(\mathrm{S}_{\infty}\right)$ of $42 \mathrm{kphotons} / \mathrm{s}$ (see Supporting Information Figure S2). Above saturation the quantum dot approaches one photon emitted per excitation pulse, that is, $2.5 \mathrm{Mphotons} / \mathrm{s}$, given our laser repetition rate of $2.5 \mathrm{MHz}$, limited by the less than unitary 
quantum efficiency $(\sim 0.7$, as provided by the manufacturer, Invitrogen) and by the off-states of the quantum dot $(\sim 30 \%$ on-state due to blinking) to a value of $\sim 0.5 \mathrm{Mphotons} / \mathrm{s}$.

Momentum spectroscopy of the emitted light directly accesses the coupling of the emitter to the nanofiber modes and allows us to calculate the modal coupling in an original way, without measuring the light transported by the nanofiber. Here, we apply Fourier patterns analysis which is emerging as a reliable quantitative tool to probe otherwise inaccessible information about the emitter ${ }^{33}$ and its emission directionality. ${ }^{11}$ We measure the light emitted in momentum space by recording the angular patterns of the radiation emerging from a quantum dot inside a nanofiber lying on glass. Index matching enables access to large angles, up to the 1.45 numerical aperture (NA) of our objective, which encompasses the wave-vectors of the guided modes beyond the air light-line. We collect Fourier patterns as shown in Figure $2 \mathrm{a}$ and $\mathrm{b}$ for two nanofibers: $\mathrm{a}$ thin one, in the single mode regime $(D / \lambda=0.5$, Figure $2 \mathrm{a})$ and a thick one, in the multimode regime $(D / \lambda=0.9$, Figure $2 b)$. Each pair of momenta lobes (positive and negative $k_{x}$ where $x$ is the direction of the longitudinal axis of the nanofiber) beyond the air light-line $\left(\left|k_{x}\right|>1\right)$, elongated in a direction orthogonal to the nanofiber, is the $k$-vector distribution of the distinct nanofiber modes. This is a clear experimental signature of the individual coupling of the quantum dot to each of the nanofiber modes, which is in very good agreement with the calculated Fourier patterns shown in Figure $2 \mathrm{c}$ and $\mathrm{d}$.

Figure 2e plots the mode dispersion structure reconstructed from the calculated angular pattern of a dipolar source in the center of a nanofiber (see Methods). The intensity map indicates the coupling strength of a longitudinal (blue) and transverse (red) dipole to the nanofiber, normalized to unity, which is the maximal value in the plot. The guided modes are visible as two lines that stem from the air light-line $\omega=c_{0} k_{x}$ growing to larger $k$-vectors up to the polymer light-line $\omega=$ $c_{0} k_{x} / n$, where $\omega$ is the light frequency, $c_{0}$ the speed of light in vacuum, and $n$ the refractive index. Because of the different spatial symmetry of the modes, the transverse (red) dipole couples almost exclusively to the fundamental mode $\mathrm{LP}_{01}$, and the longitudinal (blue) to the second higher mode $\mathrm{LP}_{11}$. Instead, the light emitted inside the light cone in air $\left(\omega>c_{0} k_{x}\right)$ corresponds to uncoupled radiation, emitted into free-space modes. We confirm this selective coupling and the nature of the modes by superimposing the analytical solution (dashed white lines) of the dispersion relations of the modes for a freestanding nanofiber. ${ }^{34}$ The analytical solution matches the maxima of the coupling of the two dipole-orientations which confirms that the strength of the momenta peak is a direct measure of the coupling of the emitter to a specific wave-vector, that is, to a specific nanofiber mode.

Coupling of an emitter to the propagating waveguide modes is required for transport and manipulation of the emitted single photons. Plasmonic nanostructures can offer very high coupling efficiencies, as high as $21 \%$ for silver nanowires ${ }^{14}$ or $42 \%$ for Vgroove channels, ${ }^{16}$ although propagation of coupled photons is limited by the strong Ohmic losses in the metal. Instead, dielectrics can offer much longer propagation length at the price of a weaker coupling, which has been measured at roomtemperature to reach $\sim 30 \%$ for quantum dots outside a silica nanofiber, approaching experimentally ${ }^{5,29}$ the theoretical limit imposed by evanescence coupling. ${ }^{35}$ Higher values, close to $100 \%$, can be reached at cryogenic conditions for the coupling of epitaxial quantum dots to photonic crystal waveguides ${ }^{31}$

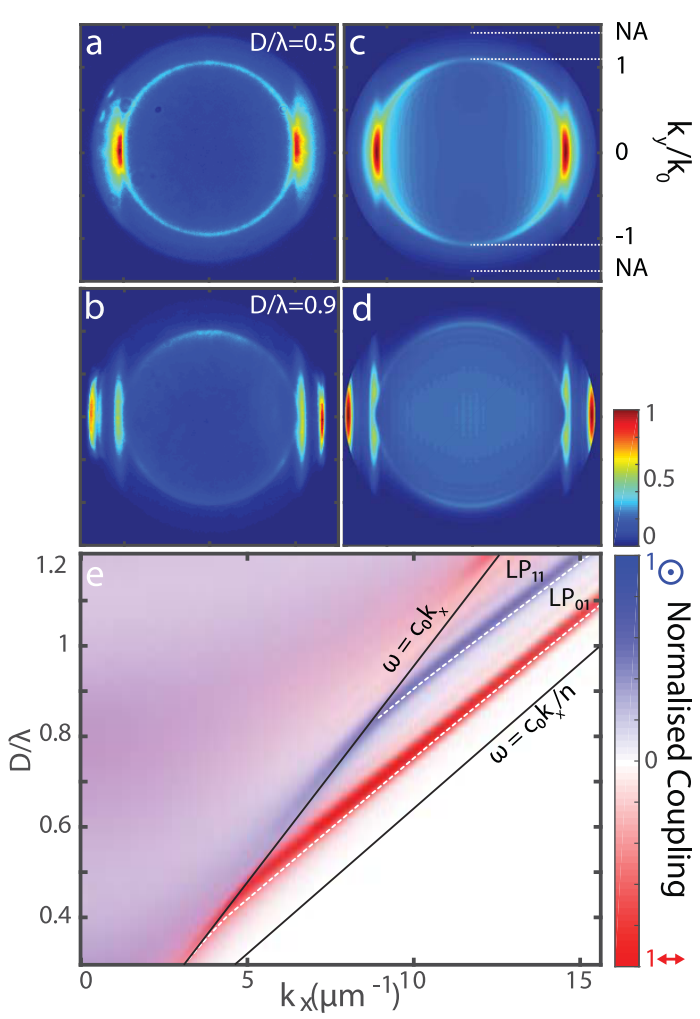

Figure 2. Momentum spectroscopy analysis. (a), (b) Experimental angular patterns and, (c), (d), the corresponding theoretical calculations (FDTD) of the radiation emitted from a quantum dot in nanofibers on glass of different diameter for $D / \lambda=0.5(\mathrm{a}-\mathrm{c})$ for $D / \lambda=0.9(\mathrm{~b}-\mathrm{d}) ; k_{0}$ is the light wavevector in air. (e) Theoretical calculations of the coupling of a dipole to the modes of the nanofiber obtained from the angular emission patterns of a longitudinal (blue) and transverse (red) dipole located at the center of the nanofiber, normalized to the maximum coupling (see Supporting Informations). The light line in air and in the polymer is indicated by the black lines, delimiting the region with guided modes. Inside the air light-cone, the light corresponds to uncoupled emission and is therefore similar for both dipolar orientations. Instead, the emission for k-vectors beyond the air light line can couple to the nanofiber modes (high intensity red and blue bands). The emission maxima correspond to the dispersion of the two nanofiber modes, proving that for reasons of symmetry the transverse dipole couples exclusively to the $L_{P_{01}}$, and the longitudinal dipole to $L P_{11}$. The analytical dispersion relation of the first two modes of the nanofiber in air $\left(\mathbf{L P}_{01}\right.$ and $\left.\mathbf{L P}_{11}\right)$ are plotted as white dotted lines.

although this is limited to narrow-band resonance, or $\sim 18 \%$ for single molecules inside a broadband pulled glass capillary. ${ }^{30}$

Here, we developed a different technique based on the momentum spectroscopy of the emitted light; from the ratio of the coupled and uncoupled light, the total coupling constant $\beta$ as well as the coupling to the fundamental mode $\beta_{01}$ can be estimated. The coupling efficiency $\beta$ is obtained as the ratio between the light emitted into the guided modes region $\left(\left|k_{x}\right|>\right.$ 1) and the total emitted light $\beta=I\left(\left|k_{x}\right|>1\right) / I$, whereas $\beta_{01}$ is the ratio between the intensity of the k-vector corresponding to the mode $\mathrm{LP}_{01}$ and the total emitted light (see Methods). The obtained coupling values are shown in Figure 3, where the lines represent the theoretical predictions and the points indicate the experimental data. Although the total coupling $\beta$ remains roughly constant for all diameters (see Supporting Information 


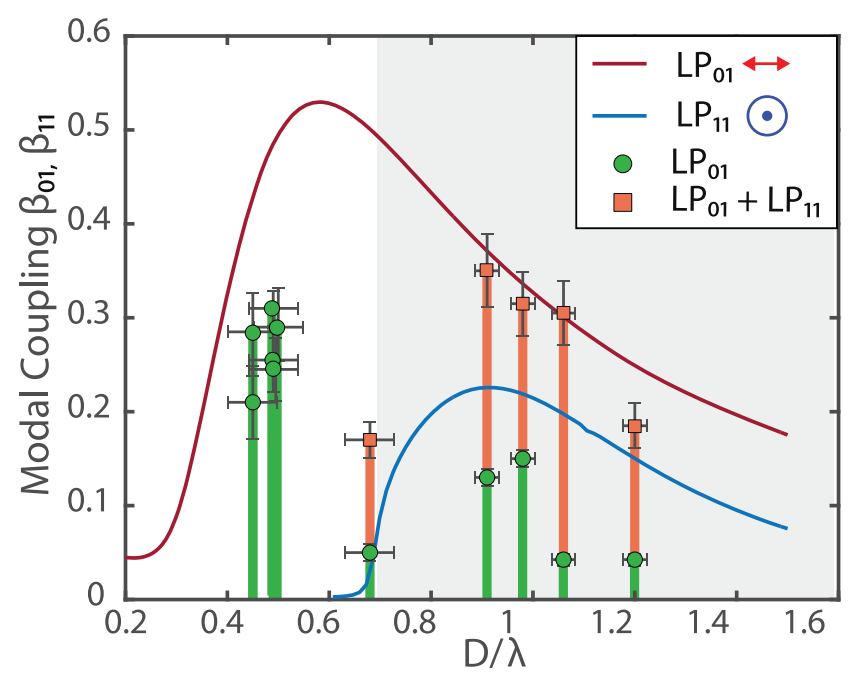

Figure 3. Modal coupling of the quantum dot to the nanofiber. Estimation of the emitter-nanofiber coupling via momentum spectroscopy. The experimental values for the coupling to the fundamental mode $\mathrm{LP}_{01}$ are plotted as circular green points (and green bar). In the multimode region (gray shaded area), coupling to the second higher mode $L P_{11}$ are shown by an orange bar and the total coupling is marked by orange squares. The experimental values are compared to the theoretical calculations for the two dipole-orientations (red line for transverse, and blue line for longitudinal). The coupling to the fundamental mode $\beta_{01}$ quickly drops for $D \geq 0.7 \lambda$ due to the emergence of other modes. The error bars originate from the uncertainty in the position of the peaks in the $k$-space.

Figure S3), the theoretical coupling $\beta_{01}$ to the fundamental mode (red line in Figure 3) reaches its maximum value of $\beta_{01}=$ $53 \%$ at $D / \lambda=0.55$, and then drops for larger diameters as more modes become available. The measured coupling values (green bars in Figure 3$)$ are in the range $\beta_{01}=(21 \pm 4) \%-(31 \pm 2) \%$ for single mode nanofibers, and $\beta_{01}=(5 \pm 1) \%-(15 \pm 1) \%$ for multimode nanofibers, where also the coupling to the second higher mode $\mathrm{LP}_{11}$ increases (orange bars in Figure 3 ). The errors come from the uncertainty in the position of the peaks in the $k$-space. The accuracy of this estimation can be confirmed by FDTD calculations that show an agreement within $10 \%$ between the values of $\beta$ obtained from the two methods of momentum spectroscopy and direct modal coupling (see Supporting Information and Figure S4). As expected, the experimental values are lower than the theoretical prediction for a transverse or longitudinal dipole, as the quantum dot's dipole moment is randomly oriented in the experiments. These findings confirm that large single mode coupling can only be achieved with subwavelength nanofibers. The momentum spectroscopy method here developed is also ideal for probing nanofibers with more complex modes, as for example periodically corrugated nanofibers, ${ }^{36}$ where the induced bandgaps would be evident in the dispersion relation.

When the nanofiber is intentionally doped with many quantum dots, their fluorescence at location distant from the illumination spot is a direct evidence of transport of the exciting laser light through the nanofiber, as shown in Figure 4a, which shows quantum dots excited as far as $60 \mu \mathrm{m}$ from the laser spot. The granularity of the quantum dot doping prevents a quantitative estimation of the propagation length, which instead can be done by recording how the fluorescence intensity leaks from a nanofibers homogeneously doped with a near-infrared
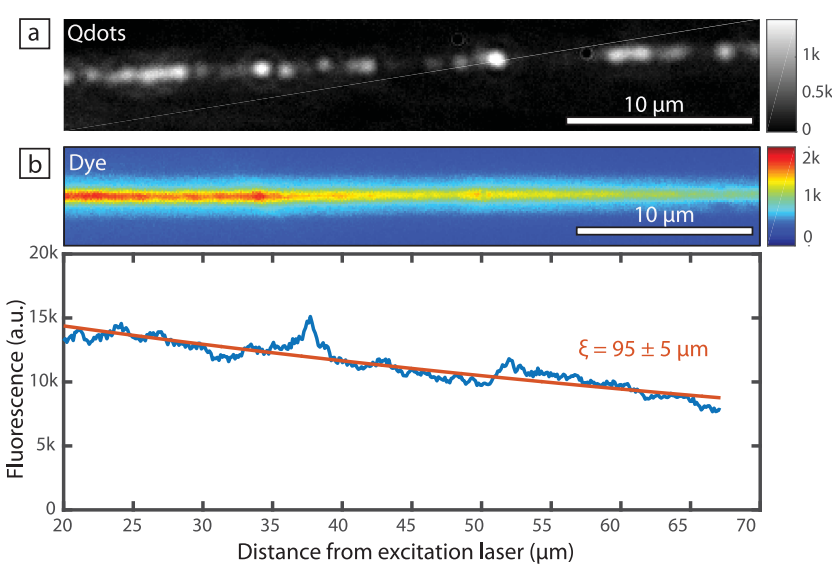

Figure 4. Light transport through the nanofiber. Panel (a) shows a nanofiber with an intentional high-density of quantum dots when remotely excited. The excitation position is outside the field of view. The laser beam couples to the nanofiber and is transported through it, which is revealed by the quantum dots excited distant locations. (b) More quantitative measure of the transport length can be obtained by exciting a nanofiber doped with an IR dye emitting around $890 \mathrm{~nm}$. The excitation is $20 \mu \mathrm{m}$ outside the field of view. The fluorescence intensity recorded along the nanofiber is fitted with and an exponential function $[A \exp (-x / \xi)]$ after subtracting the background, obtaining a propagation length of $\xi$ $=(95 \pm 5) \mu \mathrm{m}$.

dye, as shown in Figure 4b. By fitting with an exponential model the leaked light, we obtain an optical loss coefficient $\xi^{-1}$ $=(105 \pm 5) \mathrm{cm}^{-1}$, corresponding to a propagation length $\xi=$ (95 \pm 5$) \mu \mathrm{m}$. Both the nanofiber scattering and the dye reabsorption $\left(\sim 58 \mathrm{~cm}^{-1}\right.$, from bulk absorption measurements) are involved in determining the resulting $\xi$ value. Theoretically, for a nanofiber in air we expect a propagation length of 100$1000 \mu \mathrm{m}$, as calculated by including Rayleigh scattering from the nanofiber surface roughness (a few nanometers) ${ }^{37}$ as measured by atomic force microscopy.

As both long propagation and large coupling are important criteria to assess the quality of an hybrid nanoscale architecture, the most recent figure of merit (FOM) introduced by Bermúdez-Ureña et al. ${ }^{16}$ combines them in the product $(\beta P$ $\xi) / \lambda$, of mode coupling $(\beta)$, Purcell factor $(P)$, and propagation length $(\xi)$ normalized by free-space operating wavelength $(\lambda)$. We suggest here that $\beta$ is replaced by the modal coupling, that is, $\beta_{01}$ for the fundamental mode, which has more practical importance for single-mode operation. The state-of-the-art in plasmonic systems is reported to be FOM $=6.6 \pm 23 \%$, whereas for our dielectric nanofibers, we can calculate a FOM of $44 \pm 16 \%$ coming from a coupling of $\beta_{01}=(31 \pm 2) \%$ and a Purcell factor with respect to vacuum of $1.2 \pm 15 \%$ and the propagation length $\xi=(95 \pm 5) \mu \mathrm{m}$. Although plasmonic systems can strongly engineer the Purcell factor, dielectric nanostructures, with negligible Ohmic losses, offer longer propagation lengths, resulting in a large FOM. This FOM is useful to compare nanoscale geometries, whereas it loses importance for macroscopic systems such as conventional single mode fibers for which the very long propagation length dominates the FOM.

\section{CONCLUSIONS}

In conclusion, we report on a nanofiber single-photon light source architecture, integrating a quantum emitter into a low 
loss single mode optical waveguide and operating at room temperature. Using an approach based on momentum spectroscopy, we have quantified the coupling efficiency of individual quantum dots to each nanofiber mode, obtaining broadband coupling of up to $31 \%$ of the emitted light (see also Supporting Information Figure S5) and a FOM of $44 \pm 16 \%$. Through their robustness and flexibility, these nanofibers have potential as a future platform for nanoscale quantum optics as they can be connected to form a network of coupled emitters for quantum technological applications. Moreover, these systems are compatible with plasmonic systems, and therefore suitable for further photonic engineering.

\section{METHODS}

Nanofibers Fabrication. One $\mathrm{nM}$ solution containing CdSeTe quantum dots (Invitrogen QD800, emitting at $790 \mathrm{~nm}$ ) was mixed with a chloroform solution of PMMA (MW 120 000, Sigma-Aldrich). In order to obtain submicron fibers, $30 \mathrm{mg}$ of tetrabutylammonium iodide organic salt was added to the solution. By measuring the emission spectrum of the quantum dots, we checked that the solvent, polymer matrix, and organic salt do not affect the photoluminescence properties of the embedded emitters. The background fluorescence from PMMA is estimated to be $<1 \%$ of the emission from the $\mathrm{QD}$, as determined both by measuring the background fluorescence collected from the excited spot and by exciting $10 \mu \mathrm{m}$ away from the edge of an intentionally cut nanofiber and collecting at the edge (see Supporting Information Figure S5). For light transport measurements, the IR-140 organic chromophore was added to the PMMA solution, using a concentration of $0.1 \%$ weight:weight relative to the PMMA polymer matrix. The solution was loaded in a $1 \mathrm{~mL}$ syringe tipped with a $27 \mathrm{G}$ gauge stainless steel needle and delivered at $0.5 \mathrm{~mL} \mathrm{~h}^{-1}$ constant rate by means of a syringe pump (Harvard Apparatus, Holliston, MA). A bias voltage of $8 \mathrm{kV}$ was applied between the needle and the metallic collector by a high voltage power supply (Glassman High Voltage). Glass coverslip substrates $\left(18 \times 18 \mathrm{~mm}^{2}\right)$ were placed on a $10 \times 10$ $\mathrm{cm}^{2} \mathrm{Cu}$ plate used as grounded collector, positioned $10 \mathrm{~cm}$ away from the needle. Free-standing nanofibers were obtained by deposition on a TEM grid (TAAB Laboratories Equipment Ltd.) used as collector. Electrospinning was performed with a relative humidity and temperature of about $40 \%$ and $25{ }^{\circ} \mathrm{C}$, respectively. Reference thin films on glass coverslip substrates were obtained by spin-coating the solution used for electrospinning at $6000 \mathrm{rpm}$ for $40 \mathrm{~s}$. The nanofiber morphology was investigated by SEM (FEI Nova NanoSEM 450 or Zeiss) and atomic force microscopy (Veeco Instruments). The fiber diameters were in the range of $300-1000 \mathrm{~nm}$, with a surface roughness (root-mean-square, RMS) of the order of few $\mathrm{nm}$. The fibers have almost a round-shaped cross section (Figure S6a,b), with a ratio between the height and the width in the range 0.9-1. A maximum variation of $70 \mathrm{~nm}$ of the fiber diameter over a length of $1 \mathrm{~mm}$ was measured (Figure S6c-h).

Confocal Optical Microscopy. The excited state lifetime of isolated quantum dots was measured by confocal microscopy and TCSPC (TimeHarp 260, PicoQuant) with an overall temporal resolution of $\sim 100-200$ ps. The quantum dots were excited with a $100 \mathrm{ps}, 634 \mathrm{~nm}, 2.5 \mathrm{MHz}$ repetition rate laser (average power $1.5 \mu \mathrm{W}$ ) through an air objective $(\mathrm{NA}=0.95)$ or an oil immersion objective $(\mathrm{NA}=1.45)$. The light emitted was collected by the same objective and directed to an avalanche photodiode (APD). The lifetime traces were fitted with both a single exponential and a log-normal model, and the one providing the highest accuracy was retained. Antibunching was measured through the same setup, but using two APDs in start-stop configuration. The intensity correlation function $g_{2}(t)$ measured from a quantum dot under CW excitation was fitted to $g_{2}(t)=A(1-(1 / N)$ $\left.\exp \left(|t| / \tau^{\prime}\right)\right)$. The collection efficiency of our microscope is estimated to be $\sim 5 \%$ for polymer films and $1.45 \mathrm{NA}$ oil immersion objective and $\sim 2-3 \%$ for free-standing fibers and $0.95 \mathrm{NA}$ air objective. These values are obtained as the ratios between the measured saturation intensity of several individual QDs and the expected emission intensity at saturation (laser repetition rate times the quantum efficiency). ${ }^{5}$

Optical Fourier Imaging. We recorded the angular emission patterns $I\left(k_{x}, k_{y}\right)$ of isolated quantum dots by imaging the back-focal plane into a CCD camera (Princeton Instruments, Pixis 400). The excitation and collection was achieved through an oil immersion objective $(100 \times, \mathrm{NA}=1.45)$. We calculated the intensity distribution $I\left(k_{x}\right)=\Sigma_{k_{y}} I\left(k_{x}, k_{y}\right)$, where $k_{x}$ is the component of $\vec{k}$ along the nanofiber axis. We estimated the nanofiber diameter $D$ by comparing the position of the peaks in the experimental Fourier patterns with the theoretical modal dispersion. As the nanofiber modes are only marginally affected by the glass interface, the light emitted in the $k$ regions beyond the light-cone in air corresponds to guided modes $\left(\left|k_{x}\right|\right.$ $>1$ ), and the light inside the light cone in air to the uncoupled modes. For multimodal fibers, owing to the modes' orthogonality in $k$-space, different modes have different wave vectors for the same energy; this allows assessing the individual coupling to these modes by measuring the relative strength of the Fourier peaks. In this way, the coupling to the fundamental mode $\beta_{01}$ can be separated from the total coupling $\beta$ to all the waveguide modes.

The coupling efficiency $\beta$ is obtained as the ratio between the light emitted into the guided modes region $\left(\left|k_{x}\right|>1\right)$ and the total emitted light

$$
\beta=I\left(\left|k_{x}\right|>1\right) / I
$$

while $\beta_{01}$ is the ratio between the intensity of the $k$-vector corresponding to the mode $\mathrm{LP}_{01}$ and the total emitted light. This can be generalized for all other higher-energy modes.

An uncertainty is attributed to both $D$ and $\beta$ due to the uncertainty in the $k$-space calibration that we quantify as 0.05 . The accuracy of this estimation can be confirmed by finite difference time domain (FDTD) calculations that show an agreement within $10 \%$ between the values of $\beta$ obtained from the two methods of momentum spectroscopy and direct modal coupling (See Supporting Information Figure S4).

Numerical Calculations. FDTD simulations were performed using a commercial package (Lumerical). The local density of optical states (LDOS) maps were obtained by considering the intensity emitted by a dipolar source in different position inside the nanofiber. The total coupling efficiency was obtained by calculating the intensity transmitted through a monitor crossing the free-standing nanofiber 4 $\mu \mathrm{m}$ away from the source; the modal coupling efficiency was obtained by mode projection of the same intensity onto the independently calculated nanofibers modes. The angular patterns were obtained by means of far field projections of the electromagnetic fields in a plane $300 \mathrm{~nm}$ outside the nanofiber inside the substrate of a nanofiber laying on glass. Calculations with and without the glass substrate show that the low-refractive index interface below the nanofiber only weakly affects the modal dispersion, inducing only a spectral broadening due to the increased losses into the glass.

\section{ASSOCIATED CONTENT}

\section{Supporting Information}

The Supporting Information is available free of charge on the ACS Publications website at DOI: 10.1021/acsnano.6b02057.

Purcell factor measurement, data on quantum dot saturation intensity, additional calculation of modal coupling and information about momentum spectroscopy, and data about broad band coupling of quantum dots to nanofibers and atomic force microscopy. (PDF)

\section{AUTHOR INFORMATION}

\section{Corresponding Authors}

*E-mail: dario.pisignano@unisalento.it.

*E-mail: andrea.camposeo@cnr.it.

*E-mail: riccardo.sapienza@kcl.ac.uk. 


\section{Notes}

The authors declare no competing financial interest.

\section{ACKNOWLEDGMENTS}

We wish to thank Simon Fairclough for fruitful discussions. The research leading to these results has received funding from the Engineering and Physical Sciences Research Council (EPSRC), from the European Union's Seventh Framework Programme (FP7/2007-2013) EU Project People, from the Leverhulme Trust (RPG-2014-238), from the Royal Society (Research Grant no. RG140457), and from the European Research Council under the European Union's FP/2007-2013/ERC Grant Agreement no. 306357 ("NANO-JETS"). The data is publicly available in Figshare. ${ }^{38}$

\section{REFERENCES}

(1) Chang, D. E.; Sørensen, A. S.; Demler, E. A.; Lukin, M. D. A Single-Photon Transistor Using Nanoscale Surface Plasmons. Nat. Phys. 2007, 3, 807-812.

(2) Javadi, A.; Söllner, I.; Arcari, M.; Lindskov Hansen, S.; Midolo, L.; Mahmoodian, S.; Kiršanske, G.; Pregnolato, T.; Lee, E. H.; Song, J. D.; Stobbe, S.; Lodahl, P. Single-Photon Non-Linear Optics with a Quantum Dot in a Waveguide. Nat. Commun. 2015, 6, 8655.

(3) Kumar, S.; Kristiansen, N. I.; Huck, A.; Andersen, U. L. Generation and Controlled Routing of Single Plasmons on a Chip. Nano Lett. 2014, 14, 663-669.

(4) Hwang, J.; Pototschnig, M.; Lettow, R.; Zumofen, G.; Renn; Götzinger, S.; Sandoghdar, V. A Single-Molecule Optical Transistor. Nature 2009, 460, 76-80.

(5) Fujiwara, M.; Toubaru, K.; Noda, T.; Zhao, H. Q.; Takeuchi, S. Highly Efficient Coupling of Photons from Nanoemitters into SingleMode Optical Fibers. Nano Lett. 2011, 11, 4362-4365.

(6) Heeres, R. W.; Kouwenhoven, L. P.; Zwiller, V. Quantum Interference in Plasmonic Circuits. Nat. Nanotechnol. 2013, 8, 719.

(7) Di Martino, G.; Sonnefraud, Y.; Tame, M. S.; Kéna-Cohen, S.; Dieleman, F.; Özdemir, Ş. K.; Kim, M. S.; Maier, S. A. Observation of Quantum Interference in the Plasmonic Hong-Ou-Mandel Effect. Phys. Rev. Appl. 2014, 1, 034004.

(8) De Roque, P. M.; van Hulst, N. F.; Sapienza, R. Nanophotonic Boost of Intermolecular Energy Transfer. New J. Phys. 2015, 17, 113052.

(9) Schuller, J. A.; Barnard, E. S.; Cai, W.; Chul Jun, Y.; White, J. S.; Brongersma, M. L. Plasmonics for Extreme Light Concentration and Manipulation. Nat. Mater. 2010, 9, 193.

(10) Benson, O. Assembly of Hybrid Photonic Architectures from Nanophotonic Constituents. Nature 2011, 480, 193-199.

(11) Curto, A. G.; Volpe, G.; Taminiau, T. H.; Kreuzer, M. P.; Quidant, R.; van Hulst, N. F. Unidirectional Emission of a Quantum Dot Coupled to a Nanoantenna. Science 2010, 329, 930-933.

(12) Kinkhabwala, A.; Yu, Z.; Fan, S.; Avlasevich, Y.; Müllen, K.; Moerner, W. E. Large Single-Molecule Fluorescence Enhancements Produced by a Bowtie Nanoantenna. Nat. Photonics 2009, 3, 654-657.

(13) Punj, D.; Mivelle, M.; Moparthi, S. B.; van Zanten, T. S.; Rigneault, H.; van Hulst, N. F.; García-Parajó, M. F.; Wenger, J. A Plasmonic 'Antenna-In-Box' Platform for Enhanced Single-Molecule Analysis at Micromolar Concentrations. Nat. Nanotechnol. 2013, 8, 512-516.

(14) Li, Q.; Wei, H.; Xu, H. Quantum Yield of Single Surface Plasmons Generated by a Quantum Dot Coupled with a Silver Nanowire. Nano Lett. 2015, 15, 8181-8187.

(15) Kress, S. J.; Antolinez, F. V.; Richner, P.; Jayanti, S. V.; Kim, D. K.; Prins, F.; Riedinger, A.; Fischer, M. P.; Meyer, S.; McPeak, K. M.; Poulikakos, D.; Norris, D. J. Wedge Waveguides and Resonators for Quantum Plasmonics. Nano Lett. 2015, 15, 6267-6275.

(16) Bermúdez-Ureña, E.; Gonzalez-Ballestero, C.; Geiselmann, M.; Marty, R.; Radko, I. P.; Holmgaard, T.; Alaverdyan, Y.; Moreno, E.; García-Vidal, F. J.; Bozhevolnyi, S. I.; Quidant, R. Coupling of
Individual Quantum Emitters to Channel Plasmons. Nat. Commun. 2015, 6, 7883.

(17) Caldarola, M.; Albella, P.; Cortés, E.; Rahmani, M.; Roschuk, T.; Grinblat, G.; Oulton, R. F.; Bragas, A. V.; Maier, S. Non-Plasmonic Nanoantennas for Surface Enhanced Spectroscopies with Ultra-Low Heat Conversion. Nat. Commun. 2015, 6, 7915.

(18) Kolchin, P.; Pholchai, N.; Mikkelsen, M. H.; Oh, J.; Ota, S.; Islam, M. S.; Yin, X.; Zhang, X. High Purcell Factor Due To Coupling of a Single Emitter to a Dielectric Slot Waveguide. Nano Lett. 2015, 15, 464-468.

(19) Jahani, S.; Jacob, Z. All-Dielectric Metamaterials. Nat. Nanotechnol. 2016, 11, 23-36.

(20) Chang, D. E.; Cirac, J. I.; Kimble, H. J. Self-Organization of Atoms along a Nanophotonic Waveguide. Phys. Rev. Lett. 2013, 110, 113606.

(21) Babinec, T. M.; Hausmann, B. J. M.; Khan, M.; Zhang, Y.; Maze, J. R.; Hemmer, P. R.; Lončar, M. A. Diamond Nanowire Single-Photon Source. Nat. Nanotechnol. 2010, 5, 195-199.

(22) Friedler, I.; Sauvan, C.; Hugonin, J. P.; Lalanne, P.; Claudon, J.; Gérard, J. M. Solid-State Single Photon Sources: the Nanowire Antenna. Opt. Express 2009, 17, 2095-2110.

(23) Reimer, M. E.; Bulgarini, G.; Akopian, N.; Hocevar, M.; Bavinck, M. B.; Verheijen, M. A.; Bakkers, E. P. A. M.; Kouwenhoven, L. P.; Zwiller, V. Bright Single-Photon Sources in Bottom-Up Tailored Nanowires. Nat. Commun. 2012, 3, 737.

(24) Aiello, A.; Banzer, P.; Neugebauer, M.; Leuchs, G. From Transverse Angular Momentum to Photonic Wheels. Nat. Photonics 2015, 9, 789-795.

(25) Bliokh, K. Y.; Rodríguez-Fortuño, F. J.; Nori, F.; Zayats, A. V. Spin-Orbit Interactions of Light. Nat. Photonics 2015, 9, 796-808.

(26) Persano, L.; Camposeo, A.; Pisignano, D. Active Polymer Nanofibers for Photonics, Electronics, Energy Generation and Micromechanics. Prog. Polym. Sci. 2015, 43, 48-95.

(27) Sun, D.; Chang, C.; Li, S.; Lin, L. Near-Field Electrospinning. Nano Lett. 2006, 6, 839-842.

(28) Brown, T. D.; Dalton, P. D.; Hutmacher, D. W. Direct Writing By Way of Melt Electrospinning. Adv. Mater. 2011, 23, 5651-5657.

(29) Yalla, R; Le Kien, F.; Morinaga, M.; Hakuta, K. Efficient Channeling of Fluorescence Photons from Single Quantum Dots into Guided Modes of Optical Nanofiber. Phys. Rev. Lett. 2012, 109, 063602.

(30) Faez, S.; Türschmann, P.; Haakh, H. R.; Götzinger, S.; Sandoghdar, V. Coherent Interaction of Light and Single Molecules in a Dielectric Nanoguide. Phys. Rev. Lett. 2014, 113, 213601.

(31) Arcari, M.; Söllner, I.; Javadi, A.; Hansen, S. L.; Mahmoodian, S.; Liu, J.; Thyrrestrup, H.; Lee, E. H.; Song, J. D.; Stobbe, S.; Lodahl, P. Near-Unity Coupling Efficiency of a Quantum Emitter to a Photonic Crystal Waveguide. Phys. Rev. Lett. 2014, 113, 093603.

(32) Lounis, B.; Bechtel, H. A.; Gerion, D.; Alivisatos, P.; Moerner, W. E. Photon Antibunching in Single CdSe/ZnS Quantum Dot Fluorescence. Chem. Phys. Lett. 2000, 329, 399-404.

(33) Taminiau, T. H.; Karaveli, S.; van Hulst, N. F.; Zia, R. Quantifying the Magnetic Nature of Light Emission. Nat. Commun. 2012, 3, 979.

(34) Snyder, A. W.; Love, J. Optical Waveguide Theory; Springer: New York, 2012.

(35) Klimov, V. V.; Ducloy, M. Spontaneous Emission Rate of an Excited Atom Placed Near a Nanofiber. Phys. Rev. A: At., Mol., Opt. Phys. 2004, 69, 013812.

(36) Goban, A.; Hung, C. L.; Yu, S. P.; Hood, J. D.; Muniz, J. A.; Lee, J. H.; Martin, M. J.; McClung, A. C.; Choi, K. S.; Chang, D. E.; Painter, O.; Kimble, H. J. Atom-Light Interactions in Photonic Crystals. Nat. Commun. 2014, 5, 3808.

(37) Tien, P. K. Light Waves in Thin Films and Integrated Optics. Appl. Opt. 1971, 10, 2395.

(38) Gaio, M.; Moffa, M.; Castro-Lopez, M.; Pisignano, D.; Camposeo, A.; Sapienza, R. Figshare 2016, DOI: 10.6084/m9.figshare.3407236. 